Pacific

Journal of

Mathematics

\title{
ON SOLUTIONS TO
}

COURNOT-NASH EQUILIBRIA EQUATIONS ON THE SPHERE

\author{
MICAH WARREN
}

Volume 272 No. 2

December 2014 


\title{
ON SOLUTIONS TO COURNOT-NASH EQUILIBRIA EQUATIONS ON THE SPHERE
}

\author{
MICAH WARREN
}

\begin{abstract}
We discuss equations associated to Cournot-Nash Equilibria as put forward recently by Blanchet and Carlier. These equations are related to an optimal transport problem in which the source measure is known, but the target measure is part of the problem. The resulting equation is of Monge-Ampère type with possible nonlocal terms. If the cost function is of a particular form, the equation is vulnerable to standard optimal transportation PDE techniques, with some modifications to deal with the new terms. We give some sufficient conditions for the problem on the sphere from which we can conclude that solutions are smooth.
\end{abstract}

\section{Introduction}

In this note, we discuss equations associated to Cournot-Nash equilibria as put forward in [Blanchet and Carlier 2012], a reference we henceforth abbreviate as $[B C]$. These equations are related to an optimal transport problem in which the source measure is known but the target measure is to be determined. A CournotNash equilibrium (CNE) is a special type of optimal transport: Each individual $x$ is transported to a point $T(x)$ in a way that not only minimizes the total cost of transportation, but minimizes a cost to the individual $x$ (transportation plus other). This latter cost may depend on the target distribution, and may involve congestion, isolation and geographical terms.

Blanchet and Carlier demonstrated how CNE are related to nonlinear elliptic PDEs, explicitly deriving a Euclidean version of the equation [BC, (4.6)] and showing that this problem has some very nice properties [BC, Theorem 3.8]. The fully nonlinear Monge-Ampère equation differs from "standard" optimal transport equations in that the potential itself occurs on the right-hand side, along with possibly some nonlocal terms. Here we study the problem on the sphere. Immediately one can conclude from [BC, Theorem 3.8] and [Loeper 2009] that optimal maps are

I am supported in part by NSF DMS-0901644 and NSF DMS-1161498. This work was completed while I was at Princeton University.

MSC2010: 49Q20, 91A10, 35J96.

Keywords: optimal transportation, Cournot-Nash equilibrium. 
continuous with control on the Hölder norm. We move this a step further and show that all derivative norms can be controlled in terms of the data, when the solution is smooth. When the solution is known to be differentiable enough, one can easily adapt the estimates of Ma, Trudinger and Wang [Ma et al. 2005]. To make the conclusion a priori, we must use the continuity method. Closedness follows from the same estimates, but openness is not immediate and requires some conditions. In Theorem 6 we give some conditions on the data so that the problem can be solved smoothly.

\section{Background and setup}

In this section we briefly recap the setup in [BC]. Given a space of player types $X$ endowed with a probability measure $\mu$, an action space $Y$, and a cost function

$$
\Phi: X \times Y \times \mathcal{P}(Y) \rightarrow \mathbb{R}
$$

assume $x$-type agents pay cost $\Phi(x, y, v)$ to take action $y$. Here $v \in \mathcal{P}(Y)$ is the probability measure in the action space which is the push forward of $\mu$ by the map of actions from $X$ to $Y$. Supposing that $x$-type agents know the distribution $v$, they can choose the best action $y$. A Cournot-Nash equilibrium is a joint probability distribution measure $\gamma \in \mathcal{P}(X \times Y)$ with first marginal $\mu$ such that

$$
\gamma\left\{(x, y) \in X \times Y: \Phi(x, y, v)=\min _{z \in Y} \Phi(x, z, v)\right\}=1,
$$

where $v$ is the second marginal.

We will be interested in a particular type of cost,

$$
\Phi(x, y, v)=c(x, y)+\mathcal{V}[v](y)
$$

where $c$ is the transportation cost. Lemma 2.2 of [BC] shows that a CNE will necessarily be an optimal transport pairing for the $\operatorname{cost} c$ between the measures $\mu$ and $\nu$. They further show that, if $\mathcal{V}[v]$ is the differential of a functional $\mathcal{E}[v]$, then, at a minimizer for $\mathcal{E}[\nu]+\mathcal{W}_{c}(\mu, v)$, the optimal transport will necessarily be a $\mathrm{CNE}$ (here $\mathcal{W}_{c}(\mu, v)$ is the Wasserstein distance). In particular, if the cost $\mathcal{V}_{m}[v]$ is of the form

$$
\mathcal{V}_{m}[v](y)=f\left(\frac{d v}{d m}(y)\right)+\int \phi(y, z) d v(z)+V(y),
$$

where $m$ is a "background" measure and the function $\phi(y, z)$ is symmetric on $Y \times Y$, then $\mathcal{V}_{m}$ is a differential, and a solution to the optimal transport is a CNE. (We will be licentious with notation, letting $v$ denote not only the measure, but also the density with respect to the background $m$.) From here on we suppose we are working with a solution to an optimal transport with cost $c$ between measures 
$\mu$ and $v$ which is also a CNE for a total cost $\Phi$. We also assume that the manifolds $X$ and $Y$ are compact without boundary.

One can consider the pair $\left(u, u^{*}\right)$ which maximizes the Kantorovich functional

$$
J(u, v)=\int-u d \mu+\int v d v
$$

over all $-u(x)+v(y) \leq \Phi(x, y)$. The pair $\left(u, u^{*}\right)$ will satisfy

$$
-u(x)+u^{*}(y)=\Phi(x, y)
$$

$\gamma$-almost everywhere, where $\gamma$ is the optimal measure for the Kantorovich problem. If the cost satisfies the standard Spence-Mirrlees condition (in the mathematics literature, the "twist", or [Ma et al. 2005, Section 2, condition (A1)]) we have, $\mu$-almost everywhere,

$$
-u(x)+u^{*}(T(x))=\Phi(x, T(x)) .
$$

The twist condition says that $T(x)$ is uniquely determined by

$$
T(x)=\{y: D u(x)+D c(x, y)=0\},
$$

which gives the identity

$$
D u(x)+D c(x, T(x))=0 .
$$

Note that, fixing an $x$, the quantity

$$
\Phi(x, y)-u^{*}(y)
$$

must have a minimum at $T(x)$; we conclude that

$$
D_{y} \Phi(x, T(x))=D u^{*}(T(x)) .
$$

Then by condition (2-1), for fixed $x$,

$$
\Phi(x, T(x)) \leq \Phi(x, y),
$$

which implies that

$$
D_{y} \Phi(x, T(x))=0,
$$

from which we conclude that

$$
D u^{*}(y) \equiv 0 .
$$

Now the pair $\left(u, u^{*}\right)$ is determined up to a constant. One can choose the constant in $u$ or $u^{*}$ but not both. At this point we simply choose $u^{*}=0$. Having fixed this choice, we obtain information about $u$ and the measure $v$, using (2-2) and (2-4):

$$
-u(x)=c(x, T(x))+f(v(T(x)))+\int \phi(T(x), z) d v(z)+V(T(x)) .
$$


In particular, the density $v(y)$ must be determined by

$(2-7) \quad v(T(x))$

$$
=f^{-1}\left(-u(x)-c(x, T(x))-\int \phi(T(x), T(z)) d \mu(z)-V(T(x))\right),
$$

having used the change of integration variables $T$ between $\mu$ and $v$. The optimal transportation equation (see [Ma et al. 2005]) becomes

$$
\frac{\operatorname{det}\left(u_{i j}(x)+c_{i j}(x, T(x))\right)}{\operatorname{det}\left(-c_{i s}(x, T(x))\right)}=\frac{\mu(x)}{f^{-1}(Q(x, u))} .
$$

Here and in the sequel, we use $i, j, k$ to denote derivatives in the source $X$, and $p, s, t$ to denote derivatives in the target $Y$. It will be convenient to assume that $c_{i s}$ is negative definite, which follows if we are assuming condition (A2) of [Ma et al. 2005] and have chosen an appropriate coordinate system. We will use $b_{i s}(x)=-c_{i s}(x, T(x))$. Also (to keep equations within one line) we abbreviate

$$
Q(x, u)=-u(x)-c(x, T(x))-\int \phi(T(x), T(z)) d \mu(z)-V(T(x)),
$$

with $T(x)$ being determined by (2-5).

Before we say how this fully nonlinear equation is vulnerable, we mention the "Inada-like" conditions [BC, Section 3.3]

$$
\begin{gathered}
\lim _{\nu \rightarrow 0^{+}} f(v)=-\infty \text { and } \lim _{\nu \rightarrow+\infty} f(v)=+\infty, \\
f^{\prime}>0 \text { and } f \in C^{2}\left(\mathbb{R}^{+}\right) .
\end{gathered}
$$

If $f$ satisfies these conditions, then several observations are in order. First, as noted in [BC, Theorem 3.8], on a compact manifold we get bounds away from zero and infinity for the density $v$. In the spherical distance-squared transportation cost case, this immediately gives $C^{\alpha}$-continuity of the map, by results of Loeper. Secondly, the right-hand side of (2-8) is strictly monotone in the zeroth-order term - this is crucial in obtaining existence and uniqueness results, as it will allow us to invert the linearized operator. Finally, as we will show below, the first derivatives of this density will be bounded in terms of an a priori constant (depending on the smoothness of $f$ ) and the second derivatives will be bounded by a constant times second derivatives of $u$. These estimates will allow us to take advantage of the Ma-Trudinger-Wang estimates.

We will show an estimate on smooth solutions: If a solution to (2-8) is $C^{4}$, then it enjoys estimates of all orders subject to universal bounds. In order to show that arbitrary solutions are $C^{4}$ and hence smooth, we must use a continuity method. This method relies on a linearization which requires some discussion, given the integral terms in the equation. 
The problem here, on a compact manifold with cost function satisfying the Ma-Trudinger-Wang condition, is quite simpler than the delicate boundary value problem in $[\mathrm{BC}]$. With or without the nonlocal terms, such a problem may be approached as in [Liu and Trudinger 2010]. We leave this problem aside for now.

\section{Linearization}

We take the natural log of (2-8) and then consider the functional

$$
\begin{array}{r}
F\left(x, u, D u, D^{2} u\right)=\ln \operatorname{det}\left(u_{i j}(x)+c_{i j}(x, T(x))\right)-\ln \operatorname{det}\left(b_{i s}(x, T(x))\right) \\
-\ln \mu(x)+\ln f^{-1}(Q(x, u)) ;
\end{array}
$$

the equation we want to solve is

$$
F\left(x, u, D u, D^{2} u\right)=0 .
$$

Preparing for linearization, consider (2-6) applied to $u+t v$ :

$$
D u(x)+t D \eta(x)+D c\left(x, T_{t}(x)\right)=0 .
$$

Differentiate with respect to $t$ to get

Linearizing, we obtain

$$
D \eta(x)=b_{i s}(x, T(x)) \frac{d T^{s}}{d t} .
$$

$$
L \eta=\frac{d}{d t} F(u+t \eta)=L^{0} \eta+L^{1} \eta,
$$

where

$$
\begin{aligned}
& L^{0} \eta=w^{i j} \eta_{i j}+w^{i j} c_{i j s} b^{s k} \eta_{k}+b^{i s} c_{i s p} b^{p k} \eta_{k}, \\
& \begin{aligned}
& L^{1} \eta=\frac{\left(f^{-1}(Q)\right)^{\prime}}{f^{-1}(Q)}\left(-c_{s}(x, T(x)) b^{s k} \eta_{k}-\eta-V_{s} b^{s k} \eta_{k}\right. \\
&-b^{s k} \eta_{k}(x) \int \phi_{s}(T(x), T(z)) d \mu(z) \\
&\left.-\int \phi_{\bar{s}}(T(x), T(z)) b^{s k}(z) \eta_{k}(z) d \mu(z)\right) .
\end{aligned}
\end{aligned}
$$

Here we are using

$$
w_{i j}(x)=u_{i j}(x)+c_{i j}(x, T(x)) .
$$

We note also that differentiating (2-6) shows

$$
T_{i}^{s}(x, T(x))=\frac{\partial T^{s}}{\partial x_{i}}=b^{s k}(x, T(x)) w_{k i}(x, T(x)) .
$$

We take $g_{i j}(x)=w_{i j}(x)$ to define a metric (one can check that it transforms as such), then write

$$
d \mu(x)=e^{-a(x)} d V_{g}(x),
$$


where

$$
-a(x)=\ln \mu(x)-\frac{1}{2} \ln \operatorname{det} w_{i j}(x) .
$$

From the definition of $F$ in (3-1) we have

$$
-a(x)=\frac{1}{2} \ln \operatorname{det} w_{i j}-\ln \operatorname{det} b+\ln v-F,
$$

having introduced

$$
v(x)=\ln f^{-1}(Q(x, u)) .
$$

First, we compute the weighted Laplace

$$
\triangle_{a} \eta=\triangle_{g} \eta-\nabla a \cdot \nabla \eta
$$

We begin with $\triangle_{g} \eta$, differentiating in some coordinate system (see (4-1) for very similar computations):

$$
\begin{aligned}
\frac{\left(\sqrt{\operatorname{det} w} w^{i j} \eta_{j}\right)_{i}}{\sqrt{\operatorname{det} w}} & =w^{i j} \eta_{i j}+\frac{1}{2} w^{a b} \partial_{i} w_{a b} w^{i j} \eta_{j}-w^{i a} w^{b j} \partial_{i} w_{a b} \eta_{j} \\
& =w^{i j} \eta_{i j}+w^{a b} w^{i j}\left(\partial_{i} w_{a b}-\partial_{b} w_{i a}\right) \eta_{j}-\frac{1}{2} w^{a b} \partial_{i} w_{a b} w^{i j} \eta_{j} \\
& =w^{i j} \eta_{i j}+\left(w^{b a} c_{a b s} b^{s j}-w^{i j} c_{i s k} b^{s k}\right) \eta_{j}-\frac{1}{2} w^{i j}(\ln \operatorname{det} w)_{i} \eta_{j} \\
& =L^{0} \eta-b^{i s} c_{i s p} b^{p k} \eta_{k}-w^{i j} c_{k i s} b^{s k} \eta_{j}-\frac{1}{2} w^{i j}(\ln \operatorname{det} w)_{i} \eta_{j}
\end{aligned}
$$

Thus

$$
\begin{aligned}
\triangle_{a} \eta= & L^{0} \eta-b^{i s} c_{i s p} b^{p k} \eta_{k}-w^{i j} c_{k i s} b^{s k} \eta_{j}-\frac{1}{2} w^{i j}(\ln \operatorname{det} w)_{i} \eta_{j} \\
& \quad+\frac{1}{2} w^{i j}(\ln \operatorname{det} w)_{i} \eta_{j}-w^{i j}(\ln \operatorname{det} b)_{i} \eta_{j}+(\ln v)_{i} w^{i j} \eta_{j}-F_{i} w^{i j} \eta_{j} \\
= & L^{0} v+(\ln v)_{i} w^{i j} \eta_{j}-F_{i} w^{i j} \eta_{j},
\end{aligned}
$$

and hence

$$
L \eta=\triangle_{a} \eta+L^{1} \eta-(\ln v)_{i} w^{i j} \eta_{j}+F_{i} w^{i j} \eta_{j} .
$$

Next, we compute

$$
\begin{aligned}
(\ln v)_{i}=\frac{\left(f^{-1}(Q)\right)^{\prime}}{f^{-1}(Q)}\left(-u_{i}(x)-c_{i}(\right. & x, T(x))-c_{s}(x, T(x)) b^{s k} w_{k i} \\
& \left.-b^{s k} w_{k i} \int \phi_{s}(T(x), T(z)) d \mu(z)-V_{s} b^{s k} w_{k i}\right) .
\end{aligned}
$$

Noting that $-u_{i}(x)-c_{i}(x, T(x))$ vanishes, and (3-4), we have

$$
L^{1} \eta-(\ln v)_{i} w^{i j} \eta_{j}=\frac{\left(f^{-1}(Q)\right)^{\prime}}{f^{-1}(Q)}\left(-\eta-\int \phi_{s}(T(x), T(z)) b^{s k}(z) \eta_{k}(z) d \mu(z)\right) .
$$


Next, we compute the integral term in the previous expression. Notice

$$
\begin{aligned}
\int\langle\nabla \phi(y, T(z)), \nabla \eta\rangle e^{-a(z)} d V_{g}(z) & =\int \phi_{s}(y, T(z)) b^{s k} w_{k i} \eta_{j} w^{i j} e^{-a(x)} d V_{g} \\
& =\int \phi_{s}(T(x), T(z)) b^{s k} \eta_{k}(z) d \mu(z) .
\end{aligned}
$$

Now, integrating by parts, we have

$$
-\int \phi_{s}(T(x), T(z)) b^{s k} \eta_{k}(z) d \mu(z)=\int \phi(T(x), T(z)) \triangle_{a} \eta(z) e^{-a(z)} d V_{g}(z) .
$$

Combining, we have

$$
L \eta=\triangle_{a} \eta-h(x) \eta(x)-h(x) \int \phi(T(x), T(z)) \triangle_{a} \eta(z) d \mu(z)+\langle\nabla F, \nabla \eta\rangle,
$$

using the shorthand

$$
h(x)=\frac{\left(f^{-1}(Q)\right)^{\prime}}{f^{-1}(Q)},
$$

which represents a positive differentiable quantity if $f$ satisfies (2-9). In particular, if $f(\tau)=\ln \tau$ then $h$ will be identically 1 . When $F \equiv 0$ we have the following.

Proposition 1. At a solution of (3-2), the linearized operator takes the form

$$
L \eta=\triangle_{a} \eta-h(x) \eta(x)-h(x) \int \phi(T(x), T(z)) \triangle_{a} \eta(z) d \mu(z) .
$$

Lemma 2. Suppose that

$$
\max _{(x, y) \in X \times Y} h(x)|\phi(x, y)|<1 .
$$

Then the operator (3-8) has trivial kernel.

Proof. To make use of some functional analytic formality, we define operators $A$, $J, h$, and $I$ on the space $\mathcal{B}=L^{2}(X, d \mu)$ by

$$
\begin{aligned}
& {[A \eta](x)=\triangle_{a} \eta(x),} \\
& {[J \eta](x)=\int \phi(T(x), T(z)) \eta(z) d \mu(z),} \\
& {[h \eta](x)=h(x) \eta(x),} \\
& {[I \eta](x)=\eta(x) .}
\end{aligned}
$$

Then $L=A-h-h J A=(I-h J) A-h=(I-h J)\left(A-(I-h J)^{-1} h\right)$.

First, we have the pointwise estimate

$$
\begin{aligned}
{[h J \eta](x) } & =\int h(x) \phi(T(x), T(y)) \eta(y) d \mu(y) \\
& \leq\left\|\int h(x) \phi(T(x), T(y)) d \mu(x)\right\|_{L^{2}}^{1 / 2}\|\eta\|_{L^{2}}^{1 / 2} \\
& \leq\left(\max _{(x, y) \in X \times Y} h(x)|\phi(x, y)|\right)^{1 / 2}<\|\eta\|_{L^{2}}^{1 / 2},
\end{aligned}
$$


using (3-9). Integrating this quantity over $\mu$ yields

$$
\|h J\|<1
$$

as an operator on $\mathcal{B}$, so $(I-h J)$ is invertible. Thus we have

$$
\operatorname{Ker} L=\operatorname{Ker}\left(A-(I-h J)^{-1} h\right) .
$$

Now suppose, for purposes of contradiction, that we have nontrivial $\eta \in \operatorname{Ker} L$. Then

$$
A \eta=(I-h J)^{-1} h \eta,
$$

thus

$$
\left\langle(I-h J)^{-1} h \eta, \eta\right\rangle=\langle A \eta, \eta\rangle=-\int|\nabla \eta|^{2} d \mu<0 .
$$

But, as $(I-h J)$ is invertible, we can let

$$
(I-h J) \omega=h \eta .
$$

Then

$$
\left\langle\omega, h^{-1}(I-h J) \omega\right\rangle=\left\langle(I-h J)^{-1} h \eta, \eta\right\rangle<0,
$$

that is,

$$
0>\left\langle\omega, \frac{1}{h} \omega\right\rangle-\langle\omega, J \omega\rangle \geq \frac{1}{\max h}\|\omega\|^{2}-\|J\|\|\omega\|^{2}=\left(\frac{1}{\max h}-\|J\|\right)\|\omega\|^{2},
$$

which is clearly a contradiction if $1>\max h\|J\|$.

\section{Estimates on the sphere}

From here on we specialize to the round unit sphere, with cost function half of distance squared. Note that this sphere has Riemannian volume $n \omega_{n}$.

Oscillation estimates. The following estimates are a version of [BC, Lemma 3.7]. On a compact manifold, the cost function will be bounded. Since the solution $u$ is $c$-convex, at its maximum point $x_{\max }, u$ is supported below by the cost support function $c\left(x, T\left(x_{0}\right)\right)+\lambda$. Hence, at the minimum point $x_{\min }$, we have that $u\left(x_{\min }\right) \geq c\left(x_{\min }, T\left(x_{\max }\right)\right)+\lambda$, which in turn tells us that

$$
\operatorname{osc} u \leq \operatorname{osc} c=\frac{1}{2} \pi^{2} \text {. }
$$

Next we observe that, because the integration of the density $v$ against $m$ gives a probability measure, the density $v$ must be larger than $1 /\left(n \omega_{n}\right)$ at some point $y_{0}$. Using (2-7), it follows that, at the point $x_{0}=T^{-1}\left(y_{0}\right)$,

$$
-c\left(x_{0}, y_{0}\right)-u\left(x_{0}\right)-\int \phi\left(y_{0}, T(z)\right) d \mu(z)-V\left(y_{0}\right) \geq f\left(\frac{1}{n \omega_{n}}\right),
$$


and similarly, at the point $x_{1}$ where the density $v$ is smallest,

$$
-c\left(x_{1}, y_{1}\right)-u\left(x_{1}\right)-\int \phi\left(y_{1}, T(z)\right) d \mu(z)-V\left(y_{1}\right)=f\left(v\left(x_{1}\right)\right) .
$$

Hence,

$$
\begin{array}{r}
-c\left(x_{0}, y_{0}\right)+c\left(x_{1}, y_{1}\right)-u\left(x_{0}\right)+u\left(x_{1}\right)-\int\left(\phi\left(y_{0}, T(z)\right)+\phi\left(y_{1}, T(z)\right)\right) d \mu(z) \\
-V\left(y_{0}\right)+V\left(y_{1}\right) \geq f\left(\frac{1}{n \omega_{n}}\right)-f\left(v\left(x_{1}\right)\right),
\end{array}
$$

that is,

$$
f\left(v\left(x_{1}\right)\right) \geq f\left(\frac{1}{n \omega_{n}}\right)-2 \operatorname{osc} c-2 \operatorname{osc} \phi-\operatorname{osc} V>-\infty .
$$

By Inada's conditions,

$$
v \geq f^{-1}\left(f\left(\frac{1}{n \omega_{n}}\right)-\pi^{2}-2 \operatorname{osc} \phi-\operatorname{osc} V\right)>0 .
$$

Similarly, an upper bound can be derived:

$$
v \leq f^{-1}\left(f\left(\frac{1}{n \omega_{n}}\right)+\pi^{2}+2 \operatorname{osc} \phi+\operatorname{osc} V\right)<\infty .
$$

4.1. Stayaway. Now that $v$ is under control, it follows from the stayaway estimates of [Delanoë and Loeper 2006] that the map $T(x)$ must satisfy

$$
\operatorname{dist}_{\mathbb{S}^{n}}(x, T(x)) \leq \pi-\epsilon(f, \mu, V, \phi) .
$$

In particular, the map stays clear of the cut locus. All derivatives of the cost function are now controlled.

\section{MTW estimates.}

Lemma 3. If the map $T$ is differentiable and locally invertible, then the target measure density

$$
v(T(x))=f^{-1}\left(-c(x, T(x))-u(x)-\int \phi(T(x), T(z)) d \mu(z)-V(T(x))\right)
$$

has first derivatives bounded by a universal constant and has second derivatives

$$
v_{s r}=C_{1}+C_{2 k}\left(T^{-1}\right)_{r}^{k},
$$

where the bounding constants are within a controlled range.

Proof. Differentiate in the $x_{k}$ direction:

$$
\begin{aligned}
& v_{s} T_{k}^{s}(x)=\left(f^{-1}\right)^{\prime}\left(-c_{k}(x, T(x))-c_{s}(x, T(x)) T_{k}^{s}-u_{k}\right. \\
&\left.-T_{k}^{s} \int \phi_{s}(T(x), T(z)) d \mu(z)-V_{s} T_{k}^{s}\right) \\
&=\left(f^{-1}\right)^{\prime} T_{k}^{s}(x)\left(-c_{s}(x, T(x))-\int \phi_{s}(T(x), T(z)) d \mu(z)-V_{s}(T(x))\right) .
\end{aligned}
$$


As this is true for all $k$, and $D T$ is invertible, we can conclude that

$$
v_{s}(T(x))=\left(f^{-1}\right)^{\prime}\left(-c_{s}(x, T(x))-\int \phi_{s}(T(x), T(z)) d \mu(z)-V_{s}(T(x))\right)
$$

is a bounded quantity. For second derivatives, differentiate this equation in $x$ again:

$$
\begin{array}{r}
v_{s p} T_{k}^{p}=\left(f^{-1}\right)^{\prime \prime} T_{k}^{p}(x)\left(-c_{s}(x, T(x))-\int \phi_{s}(T(x), T(z)) d \mu(z)-V_{s}(T(x))\right) \\
\times\left(-c_{p}(x, T(x))-\int \phi_{p}(T(x), T(z)) d \mu(z)-V_{p}(T(x))\right) \\
+\left(f^{-1}\right)^{\prime}\left(-c_{s k}(x, T(x))-c_{s p}(x, T(x)) T_{k}^{p}(x)\right. \\
\left.\quad-T_{k}^{p}(x) \int \phi_{p s}(T(x), T(z)) d \mu(z)-T_{k}^{p}(x) V_{s p}(T(x))\right),
\end{array}
$$

that is,

$$
\begin{array}{r}
v_{s r}=\left(f^{-1}\right)^{\prime \prime}\left(-c_{s}(x, T(x))-\int \phi_{s}(T(x), T(z)) d \mu(z)-V_{s}(T(x))\right) \\
\times\left(-c_{p}(x, T(x))-\int \phi_{p}(T(x), T(z)) d \mu(z)-V_{p}(T(x))\right) \\
+\left(f^{-1}\right)^{\prime}\left(-c_{s k}(x, T(x))\left(T^{-1}\right)_{r}^{k}-c_{s p}(x, T(x))\right. \\
\left.\quad-\int \phi_{p s}(T(x), T(z)) d \mu(z)-V_{s p}(T(x))\right) .
\end{array}
$$

Now all the terms, with the exception of the $\left(T^{-1}\right)_{r}^{k}$ term, are given by controlled constants, independent of $u$. We are done.

Before we state the main a priori estimate, we recall the Ma-Trudinger-Wang (MTW) tensor [Ma et al. 2005, p. 154]. For each $y$ in the target, one can define the MTW tensor as a $(2,2)$-tensor on $T_{x} M$ via

$$
\operatorname{MTW}_{i j}^{k l}(x, y)=\left\{\left(-c_{i j p r}+c_{i j s} c^{s m} c_{m r p}\right) c^{p k} c^{r l}\right\}(x, y) .
$$

It is by now a well-known fact that, on the sphere,

$$
\mathrm{MTW}_{i j}^{k l} \xi_{k} \xi_{l} \tau^{i} \tau^{j} \geq \delta_{n}\|\xi\|^{2}\|\tau\|^{2}
$$

for a positive $\delta_{n}$ and all vector-covector pairs such that

$$
\xi(\tau)=0 .
$$

(For more discussion of the geometry of this tensor, see [Kim and McCann 2010].)

Given a solution, we define an operator on $(2,0)$-tensors as follows. Let $h$ be a (2,0)-tensor. Given vector fields $X_{1}, X_{2}$, we define

where

$$
\left(L_{w} h\right)\left(X_{1}, X_{2}\right)=\frac{1}{\sqrt{\operatorname{det} w}} \nabla_{j}\left(\sqrt{\operatorname{det} w} w^{i j} \nabla_{i} h\right)-w^{i j} \nabla_{j} a \nabla_{i} h\left(X_{1}, X_{2}\right),
$$

$$
-a(x)=\frac{1}{2} \ln \operatorname{det} w(x)-\ln \operatorname{det} b(x)+\ln v(x, T(x))
$$


and covariant differentiation is taken with respect to the round metric.

Proposition 4. Let $u$ be a solution of (2-8). If e is a unit direction in a local chart on $S^{n}$, then

$L_{w} w(e, e)$

$\geq w^{i j}\left(-c_{i j p r}+c_{i j s} c_{k r p} c^{s k}\right) c^{p m} c^{r l} w_{m e} w_{l e}-C\left(1+\sum w^{i i} \sum w_{j j}+\sum w^{i i}+\sum w_{i i}^{2}\right)$

Proof. This was proven in the case where densities are known ahead of time by Ma et al. [2005]. Adapting their proof requires only a small modification somewhere in the middle, but for completeness (and mostly for fun), we will present the calculation.

First, we note that

$$
\begin{aligned}
& \frac{\partial_{j}\left(\sqrt{\operatorname{det} w} w^{i j}\right)}{\sqrt{\operatorname{det} w_{i j}}}-w^{i j} a_{j} \\
& =\partial_{j} w^{i j}+\frac{1}{2} w^{i j}(\ln \operatorname{det} w)_{j}+w^{i j} \frac{1}{2}(\ln \operatorname{det} w)_{j}-w^{i j}(\ln \operatorname{det} b)_{j}+w^{i j}(\ln v)_{s} T_{j}^{s} \\
& =-w^{i a} w^{b j} \partial_{j} w_{a b}+w^{i j}(\ln \operatorname{det} w)_{j}-w^{i j}\left(b^{s k} b_{s k j}+b^{s k} b_{s k t} T_{j}^{t}\right)+b^{s i}(\ln v)_{s} \\
& =-w^{i a} w^{b j}\left(\partial_{j} w_{a b}-\partial_{a} w_{b j}\right)-w^{i a} w^{b j} \partial_{a} w_{b j}+w^{i j}(\ln \operatorname{det} w)_{j} \\
& \quad-w^{i j} b^{s k} b_{s k j}-b^{t i} b^{s k} b_{s k t}+b^{s i}(\ln v)_{s} \\
& =-w^{i a} w^{b j}\left(c_{a b s} T_{j}^{s}-c_{b j s} T_{a}^{s}\right)-w^{i j} b^{s k} b_{s k j}-b^{t i} b^{s k} b_{s k t}+b^{s i}(\ln v)_{s} \\
& =b^{s i} w^{b j} c_{b j s}-b^{t i} b^{s k} b_{s k t}+b^{s i}(\ln v)_{s}
\end{aligned}
$$

using (among others) the relations

$$
\partial_{j} w_{a b}-\partial_{a} w_{b j}=c_{a b s} T_{j}^{s}-c_{b j s} T_{a}^{s}, \quad w^{b j} T_{j}^{s}=b^{s j} .
$$

Now

$$
\begin{aligned}
& L_{w} w\left(e_{1}, e_{1}\right) \\
& =\frac{1}{\sqrt{\operatorname{det} w}} \nabla_{j}\left(\sqrt{\operatorname{det} w} w^{i j} \nabla_{i} w\right)\left(e_{1}, e_{1}\right)-w^{i j} \nabla_{j} a \nabla_{i} w\left(e_{1}, e_{1}\right) \\
& =w^{i j} \nabla_{j} \nabla_{i} w\left(e_{1}, e_{1}\right)+\left(b^{s i} w^{b j} c_{b j s}-b^{t i} b^{s k} b_{s k t}+b^{s i}(\ln v)_{s}\right) \nabla_{i} w\left(e_{1}, e_{1}\right) \\
& =w^{i j}\left(\partial_{i} \partial_{j} w\left(e_{1}, e_{1}\right)-\nabla_{j} \partial_{i} w\left(e_{1}, e_{1}\right)+2 w\left(\nabla_{\nabla_{j} \partial_{i}} e_{1}, e_{1}\right)-2 \partial_{i} w\left(\nabla_{j} e_{1}, e_{1}\right)\right. \\
& \left.\quad-2 \partial_{j} w\left(\nabla_{i} e_{1}, e_{1}\right)+2 w\left(\nabla_{j} \nabla_{i} e_{1}, e_{1}\right)+2 w\left(\nabla_{i} e_{1}, \nabla_{j} e_{1}\right)\right) \\
& \quad+\left(b^{s i} w^{b j} c_{b j s}-b^{t i} b^{s k} b_{s k t}+b^{s i}(\ln v)_{s}\right)\left(\partial_{i} w\left(e_{1}, e_{1}\right)-2 w\left(\nabla_{i} e_{1}, e_{1}\right)\right) .
\end{aligned}
$$

At this point, we choose a normal coordinate system (in the round metric), then 


$$
\begin{aligned}
& L_{w} w\left(e_{1}, e_{1}\right)=\left(b^{s i} w^{b j} c_{b j s}-b^{t i} b^{s k} b_{s k t}+\right.\left.b^{s i}(\ln v)_{s}\right) \partial_{i} w\left(e_{1}, e_{1}\right) \\
&+w^{i j}\left(\partial_{i} \partial_{j} w\left(e_{1}, e_{1}\right)+2 w\left(\nabla_{j} \nabla_{i} e_{1}, e_{1}\right)\right) \\
&=\left(b^{i s} w^{b j} c_{b j s}-b^{i t} b^{s k} b_{s k t}+\right.\left.b^{i s}(\ln v)_{s}\right) \partial_{i} w_{11} \\
& \quad+w^{i j}\left(\partial_{i} \partial_{j} w_{11}-\partial_{1} \partial_{1} w_{i j}\right)+w^{i j}\left(\partial_{1} \partial_{1} w_{i j}+2 w\left(\nabla_{j} \nabla_{i} e_{1}, e_{1}\right)\right) .
\end{aligned}
$$

Again harking back to [Ma et al. 2005], we let

$$
K=C \sum w^{i i} \sum w_{j j}+C \sum w^{i i}+C \sum w_{i i}^{2}+C
$$

and note that terms of the following form are $K$ :

$K=w^{i j} T_{b}^{s}, \quad K=\left(\partial_{j} w_{i k}-\partial_{k} w_{i j}\right), \quad K=w^{i j} 2 w\left(\nabla_{j} \nabla_{i} e_{1}, e_{1}\right), \quad K=w^{i j} w_{k l} ;$

so that

$$
\begin{aligned}
L_{w} w\left(e_{1}, e_{1}\right)=-K+\left(b^{s i} w^{b j} c_{b j s}-b^{t i} b^{s k} b_{s k t}+b^{s i}(\ln v)_{s}\right) \partial_{i} w_{11} \\
+w^{i j}\left(\partial_{i} \partial_{j} w_{11}-\partial_{1} \partial_{1} w_{i j}\right)+w^{i j} \partial_{1} \partial_{1} w_{i j} .
\end{aligned}
$$

Now, differentiating

$$
\ln \operatorname{det} w_{i j}=\ln \operatorname{det} b_{i s}+\ln \mu-\ln v \text {, }
$$

we have

$$
w^{i j} \partial_{1} w_{i j}=b^{s i}\left(b_{i s 1}+b_{i s t} T_{1}^{t}\right)+(\ln \mu)_{1}-(\ln v)_{s} T_{1}^{s}
$$

and again

$$
w^{i j} \partial_{11} w_{i j}+\partial_{1} w^{i j} \partial_{1} w_{i j}=K+b^{s i} b_{i s t} T_{11}^{t}+(\ln v)_{s r} T_{1}^{r} T_{1}^{s}-(\ln v)_{s} T_{11}^{s} .
$$

Now recall Lemma 3, which gives

$$
(\ln v)_{s r} T_{1}^{r} T_{1}^{s}=\frac{C_{1 s r}+C_{2 s k}\left(T^{-1}\right)_{r}^{k}}{v} T_{1}^{r} T_{1}^{s}-(\ln v)_{s}(\ln v)_{r} T_{1}^{r} T_{1}^{s}=K ;
$$

thus

$$
w^{i j} \partial_{11} w_{i j}=w^{i a} w^{b j} \partial_{1} w_{a b} \partial_{1} w_{i j}+K+b^{s i} b_{i s t} T_{11}^{t}-(\ln v)_{s} T_{11}^{s} .
$$

Note that differentiating $T_{i}^{s}=b^{s k} w_{k i}$ yields

$$
T_{i j}^{s}=b^{s k} \partial_{j} w_{k i}-b^{s a} b^{p k} w_{k i}\left(b_{a p j}+b_{a p q} T_{j}^{q}\right),
$$

in particular

$$
T_{11}^{s}=b^{s k} \partial_{1} w_{k 1}-b^{s a} b^{p k} w_{k 1}\left(b_{a p 1}+b_{a p q} T_{1}^{q}\right) .
$$

Now it follows that

$$
\begin{aligned}
T_{11}^{s}-b^{s k} \partial_{k} w_{11} & =b^{s k}\left(\partial_{1} w_{k 1}-\partial_{k} w_{11}\right)-b^{s a} b^{p k} w_{k 1}\left(b_{a p 1}+b_{a p q} T_{1}^{q}\right) \\
& =K .
\end{aligned}
$$


Bringing in the concavity of the Monge-Ampère equation (4-5) and (4-8), we can eliminate some terms to see

$$
L_{w} w\left(e_{1}, e_{1}\right) \geq-K+b^{i s} w^{b j} c_{b j s} \partial_{i} w_{11}+w^{i j}\left(\partial_{i} \partial_{j} w_{11}-\partial_{1} \partial_{1} w_{i j}\right) .
$$

Then, using

$$
\begin{aligned}
& \partial_{1} \partial_{1} w_{i j}=u_{i j 11}+c_{i j 11}+2 c_{i j s 1} T_{1}^{s}+c_{i j s} T_{11}^{s}+c_{i j p r} T_{1}^{p} T_{1}^{r}, \\
& \partial_{i} \partial_{j} w_{11}=u_{11 i j}+c_{11 i j}+c_{11 s i} T_{j}^{s}+c_{11 s j} T_{i}^{s}+c_{11 s} T_{i j}^{s}+c_{11 p r} T_{i}^{p} T_{j}^{r},
\end{aligned}
$$

we have

$$
\begin{aligned}
& L_{w} w\left(e_{1}, e_{1}\right) \\
& \quad \geq-K+\left(b^{i s} w^{b j} c_{b j s}\right) \partial_{i} w_{11}+w^{i j}\left(c_{11 s} T_{i j}^{s}+c_{11 p r} T_{i}^{p} T_{j}^{r}-c_{i j s} T_{11}^{s}-c_{i j p r} T_{1}^{p} T_{1}^{r}\right) .
\end{aligned}
$$

From (4-6),

$$
\begin{aligned}
w^{i j} T_{i j}^{s} & =w^{i j}\left(b^{s k} \partial_{j} w_{k i}-b^{s a} b^{p k} w_{k i}\left(b_{a p j}+b_{a p q} T_{j}^{q}\right)\right) \\
& =w^{i j} b^{s k}\left(\partial_{j} w_{k i}-\partial_{k} w_{i j}+\partial_{k} w_{i j}\right)-b^{s a} b^{p j}\left(b_{a p j}+b_{a p q} T_{j}^{q}\right) \\
& =K+b^{s k} \partial_{k}(\ln \operatorname{det} w) \\
& =K
\end{aligned}
$$

by (4-4). Using (4-7) we conclude

$$
L_{w} w\left(e_{1}, e_{1}\right) \geq-K-w^{b j} c_{b j s} b^{s a} b^{p k} w_{k 1} b_{a p q} T_{1}^{q}-w^{i j} c_{i j p r} T_{1}^{p} T_{1}^{r},
$$

which is the desired result after reindexing.

Corollary 5. Second derivatives of $u$ are uniformly bounded.

Proof. Given the maximum principle estimate, this proof is standard, following [Ma et al. 2005]. For some more details in the setting of Riemannian manifolds see [Kim et al. 2012, Theorem 3.5].

\section{Main theorem}

In order to make a precise statement, we define

$$
\begin{aligned}
& v_{\text {lower }}=f^{-1}\left(f\left(\frac{1}{n \omega_{n}}\right)-2 \operatorname{osc} c-2\|\phi\|_{\infty}-\operatorname{osc} V\right) \\
& v_{\text {upper }}=f^{-1}\left(f\left(\frac{1}{n \omega_{n}}\right)+2 \operatorname{osc} c+2\|\phi\|_{\infty}+\operatorname{osc} V\right) .
\end{aligned}
$$

Similarly, an upper bound can be defined by

$$
h_{\max }=\sup _{Q \in\left[v_{\text {lower }}, \nu_{\text {upper }}\right]} \frac{\left(f^{-1}(Q)\right)^{\prime}}{f^{-1}(Q)} .
$$


Theorem 6. Suppose that $f$ satisfies the Inada-like conditions (2-9), $\mu$ and $m$ are smooth, and $\phi$ and $V$ are Lipschitz. If

$$
\max _{x, y \in M}|\phi(x, y)|<\frac{1}{h_{\max }},
$$

then there exists a smooth solution to (3-2).

Proof. For existence, we proceed by continuity [Gilbarg and Trudinger 2001, Theorem 17.6] on (3-2), letting

$$
\begin{array}{r}
F\left(t, x, u, D u, D^{2} u\right) \\
=\ln \operatorname{det}\left(D^{2} u+D^{2} c(x, T(x))\right)-\ln \operatorname{det}(-D \bar{D} c(x, T(x))) \\
-\ln (t \mu(x)+(1-t) m(x))+\ln f^{-1}(Q(t, x, T(x))),
\end{array}
$$

where

$$
Q(t, x, T(x))=-u(x)-c(x, T(x))-t \int \phi(T(x), T(z)) d \mu(z)-t V(T(x)) .
$$

At time $t=0$, a solution is given by $u \equiv 0$ : this maps the measure $m$ to itself via the identity mapping. Thus the interval $\mathcal{I}$ of $t$ for which a solution exists is nonempty. Notice that the form of (5-2) is the same as of (3-2) up to a scale of the functions $\phi$ and $V$ and a change of measure, so the estimates from the previous section all hold. From the theory of Krylov and Evans one can obtain $C^{2, \alpha}$ estimates. Thus $\mathcal{I}$ is closed. Lemma 2 with these conditions gives openness, noting that on the sphere a Laplacian has index zero, and that the linearized operator which has the same principal symbol has index zero as well.

Remark. For uniqueness, the standard PDE trick does not work immediately, even under assumptions such as those in the theorem. One may be tempted to use the standard argument [Gilbarg and Trudinger 2001, Theorem 17.1] to obtain a contradiction. However, the intermediate linearized operator will have the additional $\nabla F$ term that arises in (3-7) because combinations of $u$ and $v$ are not solutions. Our proof of invertibility fails for these, so we have no reason to expect that the proof would remain valid after being integrated. Uniqueness may be more easily obtained from geometric consideration as in [BC, Section 4]; see also [Villani 2009, Chapters 15 and 16].

However, if the integral term is not present, we can use the argument [Gilbarg and Trudinger 2001, Theorem 17.1], making the important note that on the sphere the set of $c$-convex functions is convex [Figalli et al. 2011, Theorem 3.2]. In this case, invertibility of the linearized operator follows easily from standard maximum principle arguments. 


\section{References}

[Blanchet and Carlier 2012] A. Blanchet and G. Carlier, "Optimal transport and Cournot-Nash equilibria", preprint, 2012. arXiv 1206.6571

[Delanoë and Loeper 2006] P. Delanoë and G. Loeper, "Gradient estimates for potentials of invertible gradient-mappings on the sphere", Calc. Var. Partial Differential Equations 26:3 (2006), 297-311. MR 2007b:35108 Zbl 1136.35358

[Figalli et al. 2011] A. Figalli, Y.-H. Kim, and R. J. McCann, "When is multidimensional screening a convex program?”, J. Econom. Theory 146:2 (2011), 454-478. MR 2888826 Zbl 05882918

[Gilbarg and Trudinger 2001] D. Gilbarg and N. S. Trudinger, Elliptic partial differential equations of second order, Springer, Berlin, 2001. MR 2001k:35004 Zbl 1042.35002

[Kim and McCann 2010] Y.-H. Kim and R. J. McCann, "Continuity, curvature, and the general covariance of optimal transportation”, J. Eur. Math. Soc. (JEMS) 12:4 (2010), 1009-1040. MR 2011f:49071 Zbl 1191.49046

[Kim et al. 2012] Y.-H. Kim, J. Streets, and M. Warren, "Parabolic optimal transport equations on manifolds", Int. Math. Res. Not. 2012:19 (2012), 4325-4350. MR 2981711 Zbl 1253.49036

[Liu and Trudinger 2010] J. Liu and N. S. Trudinger, "On Pogorelov estimates for Monge-Ampère type equations", Discrete Contin. Dyn. Syst. 28 (2010), 1121-1135. MR 2011e:35103 Zbl 05760053

[Loeper 2009] G. Loeper, "On the regularity of solutions of optimal transportation problems", Acta Math. 202:2 (2009), 241-283. MR 2010c:49084 Zbl 1219.49038

[Ma et al. 2005] X.-N. Ma, N. S. Trudinger, and X.-J. Wang, "Regularity of potential functions of the optimal transportation problem", Arch. Ration. Mech. Anal. 177 (2005), 151-183. MR 2006m:35105 Zbl 1072.49035

[Villani 2009] C. Villani, Optimal transport: Old and new, Grundlehren der Mathematischen Wissenschaften 338, Springer, Berlin, 2009. MR 2010f:49001 Zbl 1156.53003

Received October 4, 2013.

MICAH WARREN

DEPARTMENT OF MATHEMATICS

UNIVERSITY OF OREGON

EUGENE, OR 97403-1222

UNITED STATES

micahw@uoregon.edu 


\title{
PACIFIC JOURNAL OF MATHEMATICS
}

\author{
msp.org/pjm
}

Founded in 1951 by E. F. Beckenbach (1906-1982) and F. Wolf (1904-1989)

\section{EDITORS}

Don Blasius (Managing Editor)

Department of Mathematics

University of California

Los Angeles, CA 90095-1555

blasius@math.ucla.edu

\author{
Paul Balmer \\ Department of Mathematics \\ University of California \\ Los Angeles, CA 90095-1555 \\ balmer@math.ucla.edu \\ Robert Finn \\ Department of Mathematics \\ Stanford University \\ Stanford, CA 94305-2125 \\ finn@math.stanford.edu \\ Sorin Popa \\ Department of Mathematics \\ University of California \\ Los Angeles, CA 90095-1555 \\ popa@math.ucla.edu
}

\author{
Vyjayanthi Chari \\ Department of Mathematics \\ University of California \\ Riverside, CA 92521-0135 \\ chari@math.ucr.edu \\ Kefeng Liu \\ Department of Mathematics \\ University of California \\ Los Angeles, CA 90095-1555 \\ liu@math.ucla.edu \\ Jie Qing \\ Department of Mathematics \\ University of California \\ Santa Cruz, CA 95064 \\ qing@ cats.ucsc.edu
}

\section{PRODUCTION}

Silvio Levy, Scientific Editor, production@msp.org

\section{SUPPORTING INSTITUTIONS}

ACADEMIA SINICA, TAIPEI

CALIFORNIA INST. OF TECHNOLOGY

INST. DE MATEMÁTICA PURA E APLICADA

KEIO UNIVERSITY

MATH. SCIENCES RESEARCH INSTITUTE

NEW MEXICO STATE UNIV.

OREGON STATE UNIV.

\author{
STANFORD UNIVERSITY \\ UNIV. OF BRITISH COLUMBIA \\ UNIV. OF CALIFORNIA, BERKELEY \\ UNIV. OF CALIFORNIA, DAVIS \\ UNIV. OF CALIFORNIA, LOS ANGELES \\ UNIV. OF CALIFORNIA, RIVERSIDE \\ UNIV. OF CALIFORNIA, SAN DIEGO \\ UNIV. OF CALIF., SANTA BARBARA
}

\author{
Daryl Cooper \\ Department of Mathematics \\ University of California \\ Santa Barbara, CA 93106-3080 \\ cooper@math.ucsb.edu \\ Jiang-Hua Lu \\ Department of Mathematics \\ The University of Hong Kong \\ Pokfulam Rd., Hong Kong \\ jhlu@maths.hku.hk \\ Paul Yang \\ Department of Mathematics \\ Princeton University \\ Princeton NJ 08544-1000 \\ yang@math.princeton.edu
}

These supporting institutions contribute to the cost of publication of this Journal, but they are not owners or publishers and have no responsibility for its contents or policies.

See inside back cover or msp.org/pjm for submission instructions.

The subscription price for 2014 is US $\$ 410 /$ year for the electronic version, and \$535/year for print and electronic.

Subscriptions, requests for back issues and changes of subscribers address should be sent to Pacific Journal of Mathematics, P.O. Box 4163, Berkeley, CA 94704-0163, U.S.A. The Pacific Journal of Mathematics is indexed by Mathematical Reviews, Zentralblatt MATH, PASCAL CNRS Index, Referativnyi Zhurnal, Current Mathematical Publications and Web of Knowledge (Science Citation Index).

The Pacific Journal of Mathematics (ISSN 0030-8730) at the University of California, c/o Department of Mathematics, 798 Evans Hall \#3840, Berkeley, CA 94720-3840, is published twelve times a year. Periodical rate postage paid at Berkeley, CA 94704, and additional mailing offices. POSTMASTER: send address changes to Pacific Journal of Mathematics, P.O. Box 4163, Berkeley, CA 94704-0163.

PJM peer review and production are managed by EditFLOW ${ }^{\circledR}$ from Mathematical Sciences Publishers.

\section{PUBLISHED BY}

\section{mathematical sciences publishers \\ nonprofit scientific publishing}

http://msp.org/

(C) 2014 Mathematical Sciences Publishers 


\section{PACIFIC JOURNAL OF MATHEMATICS}

Volume $272 \quad$ No. $2 \quad$ December 2014

Marginally trapped submanifolds in space forms with arbitrary

signature

HENRI ANCIAUX

One line complex Kleinian groups

275

Waldemar Barrera, Angel Cano and JuAn Pablo

NAVARRETE

A note on flux integrals over smooth regular domains

305

IDO BRIGHT and JOHN M. LEE

On stable commutator length in hyperelliptic mapping class groups

323

DANny Calegari, NaOyuKi Monden and Masatoshi Sato

Compositions of birational endomorphisms of the affine plane

PierRetTe CASSOU-NoguÈs and DANIEl DAigle

The Bochner formula for isometric immersions

395

ALESSANDRO SAVO

On solutions to Cournot-Nash equilibria equations on the sphere

423

MICAH WARREN

Double and triple Givental's J-functions for stable quotients invariants

439

ALEKSEY ZiNGER

Acknowledgement 